\title{
REVIEW
}

For reprint orders, please contact: reprints@futuremedicine.com

\section{Personalized treatment is better than one treatment fits all in the management of patients with mCRC: a consensus statement}

\begin{abstract}
Suayib Yalcin ${ }^{*, 1}$, Diaeddine Trad², Yasser Abdel Kader ${ }^{3}$, Hafez Halawani ${ }^{4}$, Osman Gokhan Demir ${ }^{5}$, Riaz Mall ${ }^{6}$, Andrey Meshcheryakov ${ }^{7}$, Fadi Nasr ${ }^{8}$, Adam Nosworthy ${ }^{9}$, Dmitry Osinsky ${ }^{10}$, Assel Tumanova ${ }^{11}$, Serdar Turhal ${ }^{12}$, Sabine Tejpar ${ }^{13} \&$ Claus-Henning Köhne ${ }^{14}$
\end{abstract}

\begin{abstract}
The VEGF- (bevacizumab) and EGFR- (cetuximab and panitumumab) targeting monoclonal antibodies have become integral components of the first-line treatment strategies for patients with metastatic colorectal cancer (mCRC). Increasingly combination chemotherapy, with or without a targeted agent, is being used to facilitate curative liver resection and improve survival rates in patients with initially unresectable but potentially resectable $m C R C$. Currently, the only selective marker for the treatment of patients with $\mathrm{mCRC}$ is tumor RAS mutational status. BRAF status is a strong prognostic indicator. Medical and clinical oncologists from Central Asia, Russia, the Middle East, Africa and Turkey reviewed data for the use of targeted agents in the treatment of patients with $\mathrm{mCRC}$ and have formed recommendations for the biological of choice first-line for patients with $\mathrm{mCRC}$.
\end{abstract}

\section{Introduction}

Colorectal cancer (CRC) is one of the most commonly diagnosed cancers in Europe and worldwide [1,2]. In 2008, 1.23 million new cases of CRC were diagnosed and over 600,000 CRC-related deaths occurred worldwide [2]. The optimal treatment strategy for patients with clearly unresectable metastatic CRC (mCRC) has changed markedly over the last decade. A multivariate analysis of 3825 patients with mCRC treated with 5-FU-based therapy in 19 prospective randomized and three Phase II trials highlighted a heterogeneity in survival rates, which was attributed to differences in the baseline characteristics of the patients [3]. This was subsequently confirmed for patients with $\mathrm{mCRC}$ receiving oxaliplatin- and irinotecan-containing regimens $[4,5]$.

'Department of Medical Oncology, Hacettepe University Institute of Cancer, Hacettepe University, Ankara, Turkey

${ }^{2}$ Medical Oncology, Tawam Hospital, Al Ain, United Arab Emirates

${ }^{3}$ Oncology Department, Kasr Al-Ainy Oncology Center, Cairo, Egypt

${ }^{4}$ Adult Medical Oncology/Hematology, King Fahad Specialist Hospital, Dammam, Saudi Arabia

${ }^{5}$ Department of Medical Oncology, European Florence Nightingale Hospital, Istanbul, Turkey

${ }^{6}$ Hopelands Cancer Centre, Overport, Durban, South Africa

${ }^{7}$ N.N. Blokhin Cancer Research Center, Russian Academy of Medical Sciences, Moscow, Russia

${ }^{8}$ Oncology-Hematology Department, Mount Lebanon Hospital, Beirut, Lebanon

${ }^{9}$ Medical Oncology Unit, Wits University Donald Gordon Medical Centre, Johannesburg, South Africa

${ }^{10}$ Oncology Department, Kiev City Clinical Oncological Centre, Kiev, Ukraine

"Kazakh Scientific Research Institute of Oncology \& Radiology, Almaty, Kazakhstan

${ }^{12}$ Division of Medical Oncology, Marmara University, Istanbul, Turkey

${ }^{13}$ Digestive Oncology Unit, University Hospital Gasthuisberg, Belgium

${ }^{14}$ Department of Hematology \& Oncology, Klinikum Oldenburg, Oldenburg, Germany

*Author for correspondence: Tel.: +90 312305 2910; Fax: +90 312309 2912; syalcin@hacettepe.edu.tr

\section{KEYWORDS}

- bevacizumab • cetuximab

- consensus treatment recommendations $\bullet$ first line $\bullet$ metastatic colorectal cancer $\bullet$ panitumumab - RAS mutational status 
However since not all patients and their disease are identical, treatment decisions for patients with mCRC should be strategic and based on treatment goal [6-9]. As a consequence, the current European recommendations and guidelines for the treatment of patients with mCRC are unanimous in proposing that patients with initially unresectable metastatic disease should be stratified for first-line treatment into three groups according to clinical presentation. Other factors, such as the dynamics of disease progression, prognostic clinical markers, patient comorbidities, the ability to undergo a secondary resection for their metastatic disease (if appropriate), and treatment aim must also be considered (Figure 1) [6-8].

It is now generally accepted that for patients with CRC, with metastases limited to the liver and/or lung, treatment strategies should be directed towards resectability, recognizing not only the role played by liver and/or lung surgery in improved patient outcome, and possibly cure, but also the role of systemic therapy in rendering initially unresectable liver (and lung) metastases potentially resectable $[6,8,10]$. Increasingly, not only these patients, but also those with metastases that are initially considered never likely to become resectable are being offered, whenever possible, intensive systemic therapy, with palliative intent, with a view to shrinking their tumors and alleviating their symptoms. As a consequence, an increasing number of these patients are being referred for secondary resections of their metastases. Furthermore, the approval of molecularly targeted agents directed at the epidermal growth factor receptor (EGFR) and the vascular endothelial growth factor (VEGF) signaling pathways has expanded the first-line treatment options for patients with mCRC. Thus, treatment with combination chemotherapy with a targeted agent, is increasingly being used to facilitate curative liver resection and improve survival rates in patients with initially unresectable but potentially resectable $\mathrm{mCRC}$ as well as first-line in those patients with $\mathrm{mCRC}$ whose metastases are never likely to be eligible for resection.

This document provides a summary of an advisory board panel discussion held in Istanbul, on 13 December 2012, with representatives from Egypt (1), Kazakhstan (1), Lebanon (1), Russia (1), South Africa (2), Saudi Arabia (1), Turkey (3), Ukraine (1) and the UAE (1), which reviewed the current data available for the use of targeted agents in the treatment of patients with mCRC as presented by two European oncologists, Professors Sabine Tejpar (Belgium) and Henning Köhne (Germany). Interventional therapy approaches and radiotherapy and chemoradiotherapy options for patients with rectal tumors were not part of the review. The aim of the discussion was to try and optimize the systemic treatment approaches for patients with mCRC across the region and to arrive at consensus recommendations for their treatment. It should be noted that these recommendations were updated subsequent to the meeting to take into account the requirements for expanded $R A S$

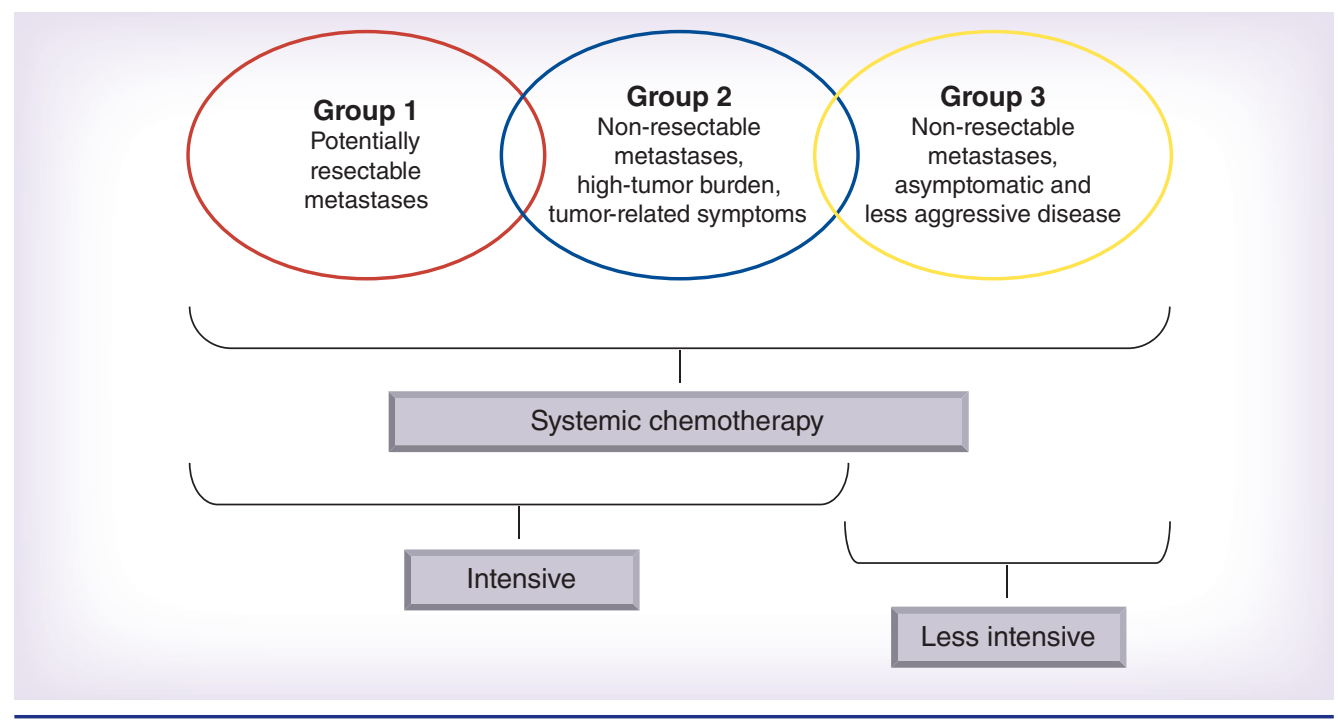

Figure 1. Patients with metastatic colorectal cancer grouped according to clinical presentation and subsequent systemic treatment. 
analysis prior to the treatment of patients with EGFR-targeted agents, with the approval of all authors.

\section{Background}

It was emphasized how little is still understood about the progression of CRC, including the heterogeneity of lesions within the individual patient. For example, 'what are the implications of tumor heterogeneity?' and 'does CRC spread from the primary tumor to the metastasis and/ or from metastasis to new metastasis?' If there are in fact two distinct metastatic processes, 'are the biological drivers for the two processes the same?' and 'what are the processes involved in disease progression while undergoing therapy?' Thus, going forward a detailed knowledge of both colorectal tumor biology and heterogeneity will be key to our understanding of which disease/signaling pathway(s) to target and when.

It is clear that we do not yet have one therapeutic approach that works for all, so currently the best we can do is identify those patients we might be able to 'cure' ( $>5$ year survival) as part of a more strategic and personalized treatment approach. Until recently, the only selective (negative) marker we had for the treatment of patients with mCRC was tumor $K R A S$ mutational status which allowed the identification and selection of the patient group who will not respond to EGFR-targeted monoclonal antibody (mAb) therapy, thus increasing the therapeutic index of EGFR-targeted therapy [11-16]. Such EGFRtargeted therapies were shown only to confer an efficacy benefit, compared with chemotherapy alone, in patients with $K R A S$ exon 2 codon $12 / 13$ wild-type (henceforth KRAS wild-type) $\mathrm{mCRC}$ treated in the first-line setting [11-16]. Subsequently, retrospective analyses of data from the PRIME and OPUS studies showed that the addition of panitumumab or cetuximab to infusional 5-fluorouracil (5-FU)/leucovorin (LV)/ oxaliplatin (FOLFOX4) may be detrimental to any patient whose tumor contained a mutation in KRAS or NRAS exons 2, 3 or $4[17,18]$. In fact, patients with tumors with $K R A S / N R A S$ mutations treated with FOLFOX4 plus panitumumab had a shorter median survival than those receiving FOLFOX4 alone [19,20], clearly suggesting that the determination of tumor $K R A S$ and NRAS mutational status should be a prerequisite of treatment with EGFR-targeted therapies plus a platinum-containing regimen. Currently, neither panitumumab nor cetuximab in combination with oxaliplatin-based chemotherapy is indicated for the treatment of CRC patients with RAS (KRAS or NRAS) mutationpositive tumors or for the treatment of those for whom the RAS status of their tumors is unknown. The PRIME study also showed $B R A F$ mutation status to have no predictive value for panitumumab $[18,20]$. $B R A F$ however, remains a powerful prognostic indicator. A similar retrospective analysis of data from the CRYSTAL study also shows that the addition of cetuximab to infusional 5-FU/LV/irinotecan (FOLFIRI) is unlikely to benefit any patient whose tumor contains any mutation in KRAS or NRAS exons 2, 3 or 4 [21].

Going forward, there is the potential for the use of other pretreatment selective markers within the established $K R A S / N R A S$ wild-type (henceforth $R A S$ wild-type) and mutant patient subsets, such as tumor BRAF and PIK3CA mutational status. However, currently these only allow the identification of small subgroups of patients and do not impact on the therapeutic strategies for the majority of patients. There is also the possibility of using the tumor shrinkage, which we know is augmented by the use of EGFR-targeted agents such as cetuximab in the treatment of patients with $R A S$ wild-type tumors, as an on-treatment marker that is predictive for outcome [22-24], as a component of a more personalized treatment approach.

Currently, the first-line treatment options that include an EGFR-targeting monoclonal antibody therapy according to defining study are:

- CRYSTAL (FOLFIRI + cetuximab) $[11,12]$;

- OPUS (FOLFOX + cetuximab) $[13,14]$;

- COIN (FOLFOX + cetuximab) [25];

- PRIME (FOLFOX + panitumumab) [15].

While, the first-line treatment options that include a VEGF-targeting agent according to study are:

- Hurwitz trial (IFL + bevacizumab) [26];

- NO16966 (XELOX/FOLFOX + bevacizumab) [27].

Bevacizumab is without doubt the therapy of choice for patients with $R A S$ mutant disease as EGFR-targeting therapies show no benefit in these patients. In addition, the efficacy of bevacizumab is known to be independent of KRAS mutation status and cross trial comparisons suggest that the efficacy is similar for cetuximab and 
bevacizumab in $K R A S$ wild-type patients. It is clear that we need further head-to head comparative studies in $K R A S / R A S$ wild-type patients such as:

- FIRE-3 (FOLFIRI + either bevacizumab or cetuximab) [28];

- PEAK (FOLFOX + either bevacizumab or panitumumab) [29];

- CALGB/SWOG 80405 (FOLFIRI/FOLFOX + either cetuximab or bevacizumab) [30].

Thus, based on the available evidence, how do we optimize the management of patients with $\mathrm{mCRC}$ and in particular those with $R A S$ wild-type disease?

\section{Optimization: the evidence}

It has been proposed, as stated previously, that the treatment decisions for all patients with $\mathrm{mCRC}$ should be strategic and based on clinical presentation and treatment goal [6-8]. In a further refinement and increased personalization of this approach, key 'STEPs' were proposed for consideration when treating patients with mCRC:

- Strategy (whether the patient should be treated with curative or palliative intent);

- Tumor biology (whether the patient presents with aggressive or indolent disease);

- EGFR ( $R A S$ mutational status of the patient's tumor(s), ie wild-type or mutant);

- Patient (age, co-morbidities, physical and psychological well-being, ability to carry out the activities of daily living).

Thus, patients with initially unresectable $\mathrm{mCRC}$ should be categorized into three groups (1-3) according to the status of their disease at presentation and tumor $R A S$ gene mutational status, and treatment goal (Figure 1). In general terms, those patients requiring an aggressive or intensive therapy approach are those group 1 patients with initially unresectable but potentially resectable metastases and those group 2 patients with clearly symptomatic disease in whom tumor regression is necessary, and those patients in whom rapid deterioration due to aggressive tumor biology and/or extensive disease is likely [7]. For group 3 patients with clearly unresectable disease with no relevant diseaserelated symptoms the options may involve a less intensive sequential therapy approach. It must be noted that only those patients in group 1 are being treated with truly curative intent.

\section{- Management of group 1 patients}

For group 1 patients, with potentially resectable liver and/or lung metastases only, who are able to tolerate intensive chemotherapy and major surgery, the treatment strategy should involve 'intensive systemic therapy,' typically combination chemotherapy plus a targeted agent. The aim of such an approach is to achieve a high response rate $(\mathrm{RR})$ with a view to rendering initially unresectable metastatic disease resectable, thus offering the patient the chance of long-term survival and potentially cure. Also, the treatment of these patients should be managed by a multidisciplinary team (MDT) as part of a more strategic and personalized treatment approach.

It is well established that standard first-line combination chemotherapy regimens can render initially unresectable colorectal liver metastases resectable [31-33]. Furthermore, resection rates are known to correlate with RR, particularly in 'selected' patients with metastatic disease limited to the liver [34]. The addition of EGFR-targeted therapies to these standard first-line combination chemotherapy regimens has been shown to confer a benefit in terms of $\mathrm{R} R$ in patients with $K R A S / R A S$ wild-type disease, in the pivotal randomized CRYSTAL, OPUS and PRIME studies $[12,14,15,17,18,21]$. In addition and of particular relevance when considering the treatment options for group 1 patients with $R A S$ wild-type disease, is the observation in the CRYSTAL study that the rate of surgery for metastases in those patients with $K R A S$ wild-type disease was higher for those patients treated with FOLFIRI plus cetuximab than for those treated with FOLFIRI alone (7.9 versus $4.6 \%$; odds ratio [OR]: 1.823 [95\% CI: $0.957-3.472] ; \mathrm{p}=0.0633)$. The corresponding $\mathrm{R} 0$-resection rates were 5.1 versus $2.0 \%$ (OR: 2.650 [95\% CI: 1.083-6.490]; $\mathrm{p}=$ $0.0265)[11,12,35]$. Similarly, in the OPUS study, the rates of metastatic surgery were significantly higher for those patients with $K R A S$ wild-type tumors who received FOLFOX4 plus cetuximab $(12.2 \%)$ than for those who received FOLFOX4 alone $(3.1 \% ; \mathrm{p}=0.0242)$. The corresponding $\mathrm{R} 0$ resection rate was more than doubled from $4.1 \%$ for those patients who received FOLFOX 4 alone to $9.8 \%$ for those who received FOLFOX 4 plus cetuximab [13]. In both the CRYSTAL and OPUS studies, the corresponding resection rates were higher for patients with KRAS wild-type 
metastatic disease confined to the liver confirming that the addition of cetuximab was still able to confer a benefit in these relatively good prognosis group 1 patients [36]. Whilst, in a Chinese trial in patients with KRAS wildtype, liver-limited disease (LLD), 18 patients $(30.5 \%)$ in the cetuximab arm and five $(8.8 \%)$ in the chemotherapy alone arm underwent an R0 resection [37]. By comparison, the data for panitumumab in the PRIME trial were less convincing with attempted secondary resection rates of 10.5 and $9.4 \%$ for FOLFOX4 plus panitumumab and FOLFOX4, respectively in patients unselected for KRAS wild-type or LLD status [15]. Interestingly, the new EPOC study showed no benefit of adding cetuximab to neoadjuvant chemotherapy for patients with resectable liver metastasis [38].

At present, cytotoxic doublets (FOLFIRI, FOLFOX or capecitabine plus oxaliplatin [XELOX (CAPOX)]) in combination with a targeted agent are considered to be acceptable options for group 1 patients requiring intensive therapy with the recommendation that cetuximab is used in combination with the standard cytotoxic doublet regimens FOLFIRI and FOLFOX, and not CAPOX or FLOX as used in the COIN and NORDIC VII trials (Table 1) $[25,39]$. The importance of the choice of chemotherapy backbone (infusional over bolus or oral fluoropyrimidine) has been confirmed in a metaanalysis of the CRYSTAL, OPUS, COIN and NORDIC VII trials [40]. Also, in this group 1 patient setting, the evidence from a meta-analysis of six randomized Phase II and III trials of oxaliplatin in combination with capecitabine or infusional 5-FU in patients with $\mathrm{mCRC}$, favors the use of FOLFOX over XELOX (CAPOX) [41]. Also, although bevacizumab could be used for 'all comers' ie patients with either $R A S$ wildtype or mutant disease, the results of the randomized NO16966 trial provide no evidence of an increase in $\mathrm{RR}$ when bevacizumab is added to FOLFOX or CAPOX [27,42]. This provides evidence favoring the use of cetuximab over bevacizumab in group 1 patients with $R A S$ wild-type disease. However the data from the BOXER [43] and OLIVIA [44] trials are more encouraging and the Hurwitz study clearly showed an increased response rate with the addition of bevacizumab to IFL [45]. The CELIM study, although not controlled for cetuximab, confirmed the RRs in patients with KRAS wild-type LLD reported for the CRYSTAL and OPUS studies, lending support to the use of cetuximab in this setting. The CELIM study also provided evidence of the clear benefit in terms of median survival for those patients undergoing resection (53.9 months) compared with those with unresectable liver disease (27.3 months) $(p=0.002)$ [46], and compares well with the results of the Chinese study in KRAS wild-type patients with LLD.

Consistent with these observations for cetuximab and resection of metastases, early tumor shrinkage (ETS) is emerging as a tool for predicting long-term outcome in patients with mCRC [47], and in particular those patients receiving EGFR antibody therapy $[23,48,49]$. Retrospective analyses of the CRYSTAL and OPUS study data have demonstrated strong associations between ETS and long-term outcomes for patients with $K R A S$ wild-type disease treated with cetuximab in combination with either FOLFIRI or FOLFOX4 [50]. Data from the CRYSTAL study show patient selection to increase median overall survival (OS) from 19.9 months for all patients [11] to 23.5 months in KRAS wild-type patients [11] to 30 months in $K R A S$ wild-type patients with $>20 \%$ tumor shrinkage at week 8 (first computed tomography [CT] scan) [22]. These data however need to be validated in other trials, particularly bevacizumab trials, where there are insufficient data [51] for bevacizumab with regards to response and tumor shrinkage, and adapted for use in routine clinical practice [22]. Recently, 'depth of response' has been proposed as a new efficacy endpoint to explain the impact of ETS on long-term outcome for EGFRtargeted therapy regimens [52-54]. Thus, these studies show that arguments in favor of using an EGFR-targeted therapy with combination chemotherapy regimens in the first-line treatment of group 1 patients with $R A S$ wild-type disease are, the quantitative benefit in terms of RR and complete (R0) resection rate, the qualitative benefit in terms of tumor shrinkage and alleviation of symptoms, and early tumor shrinkage as a predictor of clinical outcome (on-treatment marker) and guide to individual patient management.

Data from the randomized FIRE-3 study comparing FOLFIRI plus cetuximab with FOLFIRI plus bevacizumab first-line have shown a significantly better OS outcome for the cetuximab arm compared with the bevacizumab arm (28.8 vs 25.0 months, HR: 0.77, $\mathrm{p}=0.0164)$ in patients with $K R A S$ wild-type disease which increased to a difference of 7.5 months (HR: 0.70, $\mathrm{p}=0.011$ ), for $R A S$ 
Table 1. Randomized trials of EGFR antibodies as first-line treatment in KRAS wild-type metastatic colorectal cancer.

\begin{tabular}{|c|c|c|c|c|c|}
\hline Trial & Therapy & Overall response rate ${ }^{\dagger}$ & $\begin{array}{l}\text { Progression-free survival } \\
\text { (months) }^{\dagger}\end{array}$ & Overall survival (months) $^{\dagger}$ & Ref. \\
\hline $\begin{array}{l}\text { CRYSTAL } \\
(\mathrm{n}=x 666)^{\ddagger}\end{array}$ & $\begin{array}{l}\text { FOLFIRI +/- } \\
\text { cetuximab }\end{array}$ & $\begin{array}{l}57 \% \text { vs } 40 \% \S \\
\text { p }(\mathrm{CMH} \text { test })=<0.001\end{array}$ & $\begin{array}{l}9.9 \text { vs } 8.4^{\S} \\
H R=0.696 \\
p(\text { log-rank test })=0.0012\end{array}$ & $\begin{array}{l}23.5 \text { vs } 20.0^{\varsigma} \\
H R=0.796 \\
p(\text { log-rank test })=0.0093\end{array}$ & [12] \\
\hline PRIME $(n=656)^{\ddagger}$ & $\begin{array}{l}\text { FOLFOX }+/- \\
\text { panitumumab }\end{array}$ & $\begin{array}{l}55 \% \text { vs } 48 \% \\
p(\text { stratified log-rank test })= \\
0.068\end{array}$ & $\begin{array}{l}9.6 \text { vs } 8.0^{\S} \\
H R=0.80 \\
p(\text { stratified log-rank test })= \\
0.02\end{array}$ & $\begin{array}{l}23.9 \text { vs } 19.7^{\Uparrow} \\
H R=0.83 \\
p(\text { stratified log-rank test })= \\
0.072\end{array}$ & [15] \\
\hline OPUS $(n=179)^{\ddagger}$ & $\begin{array}{l}\text { FOLFOX +/- } \\
\text { cetuximab }\end{array}$ & $\begin{array}{l}57 \% \text { vs } 34 \%{ }^{5} \\
p(\text { stratified } C M H \text { test })=0.0027\end{array}$ & $\begin{array}{l}8.3 \text { vs } 7.2^{\S} \\
H R=0.567 \\
p(\text { stratified log-rank test })= \\
0.0064\end{array}$ & $\begin{array}{l}22.8 \text { vs } 18.5^{\natural} \\
H R=0.855 \\
p(\text { stratified log-rank test })= \\
0.39\end{array}$ & [14] \\
\hline $\operatorname{COIN}(n=729)^{\ddagger}$ & $\begin{array}{l}\text { XELOX/FOLFOX } \\
+/ \text { - cetuximab }\end{array}$ & $\begin{array}{l}64 \% \text { vs } 57 \%{ }^{\S} \\
p(\text { log-rank test })=0.049\end{array}$ & $\begin{array}{l}8.6 \text { vs } 8.6 \\
H R=0.96 \\
p \text { (log-rank test })=0.60\end{array}$ & $\begin{array}{l}17.9 \text { vs } 17.0 \\
H R=1.04 \\
p(\text { log-rank test })=0.67\end{array}$ & [25] \\
\hline \multicolumn{6}{|c|}{$\begin{array}{l}\text { †Chemotherapy + biologic versus chemotherapy alone. } \\
\text { †KRAS wild-type population only. } \\
\text { "Statistically significant difference. } \\
\text { 'Clinically relevant but not statistically significant difference. } \\
\text { CMH: Cochran-Mantel-Haenszel; HR: Hazard ratio. }\end{array}$} \\
\hline
\end{tabular}

wild-type patients receiving cetuximab [55], but no difference for RR (primary endpoint) and progression-free survival (PFS). Interestingly this is the only trial where RR and PFS were the same for both treatment arms with the survival benefit only being clearly demonstrated from 24 months onwards by the maintained separation of the Kaplan-Meier curves [55]. However, it should be noted that $48.2 \%$ of patients in the cetuximab arm went on to receive bevacizumab second-line and $42.9 \%$ of patients in the bevacizumab arm went on to receive cetuximab. While emerging data from the World Congress of GI Cancer showed a potentially significant improvement in tumor shrinkage and response in patients with $R A S$ wild-type disease compared with those receiving bevacizumab [56]. This observation of an increased benefit for EGFR-targeted therapy in combination with FOLFIRI in FIRE-3 is consistent with the benefit observed for FOLFOX6 plus panitumumab versus FOLFOX6 plus bevacizumab in terms of PFS and OS (although not reaching a statistically significant benefit for OS) for patients with $K R A S$ or NRAS disease wild-type for exons 2, 3 and 4 in the PEAK study [29]. These two studies suggest that the optimal treatment sequence for patients with
$R A S$ wild-type disease may be a cetuximab- or panitumumab-containing regimen first-line followed by a bevacizumab-containing regimen [28]. However, the results recently reported for the CALGB study did not confirm this [30]. The latter study showed no difference between the $K R A S$ wild-type treatment groups in terms of OS and PFS [30], and details of RR and PFS and OS for the $R A S$ wild-type patients are eagerly awaited.

However, while the evidence seems to support the use of chemotherapy plus an EGFR $\mathrm{mAb}$ therapy first-line in patients with $R A S$ wild-type disease, clearly, chemotherapy plus bevacizumab is a treatment option for group 1 patients with both $R A S$ wild-type and mutant disease, despite the fact that there are no good data for bevacizumab in combination with either irinotecan- or oxaliplatin-containing standard infusional chemotherapy regimens in patients with tumors with $R A S$ mutations. Meanwhile, FOLFOXIRI provides an alternative treatment option for patients with either $R A S$ wild-type or mutant disease as it has been shown to induce a high RR when compared with FOLFIRI alone [57] and may also be useful in combination with bevacizumab in patients with $B R A F$ mutant tumors [51,58]. 


\section{- Management of group 2 patients}

For group 2, patients with poor performance status (PS) and aggressive symptomatic disease, the treatment choice should be one that achieves a high RR and of course the best PFS and OS rates possible [6]. There is evidence that an infusional combination chemotherapy regimen should be the chemotherapy regimen of choice [59]. The combination of cetuximab with a standard infusional combination chemotherapy regimen has clearly demonstrated an improvement in symptom relief over infusional chemotherapy alone in patients with $K R A S$ wild-type disease [60]. Also, although no prospective data are available, the higher RRs and earlier response observed with cetuximab may argue in favor of using this combination for the treatment of patients with rapidly progressive $R A S$ wild-type disease. As for group 1 patients, group 2 patients with $R A S$ mutant disease could receive combination chemotherapy plus bevacizumab. However bevacizumab-containing regimens can be used for the treatment of either $R A S$ wild-type or mutant disease. Again, treatment with FOLFOXIRI plus or minus bevacizumab may be an option. In general the most effective regimen that is able to be tolerated by the patient should be used.

\section{- Management of group 3 patients}

For group 3 patients with non-resectable metastases, asymptomatic and less aggressive disease (currently 66\% of mCRC patients in Europe are treated with palliative intent) the questions for the treating physician are 'how intense should the treatment be, for how long and when should a biological be included in the therapy?'

A 'pooled/combined' analysis suggests that the addition of bevacizumab to fluoropyrimidine therapy may confer a benefit over fluoropyrimidine or irinotecan/bolus 5-FU/LV (IFL) therapy [61], while the addition of bevacizumab to the oral fluoropyrimidine capecitabine may provide some benefit in terms of PFS [62]. In the NO16966 trial, the disadvantage of CAPOX when compared with FOLFOX first-line is brought up to the level of FOLFOX in terms of efficacy outcomes by the addition of bevacizumab [27,42]. However, where more flexible dosing is possible, capecitabine being an oral fluoropyrimidine can be the preferred chemotherapy choice, especially in frailer patients and where maintainence is possible as no pumps and ports are necessary. Also, although the addition of bevacizumab to the US bolus IFL regimen, which is known to be inferior to both FOLFIRI and FOLFOX [59], confers an advantage over treatment with IFL alone there are no particularly convincing data from randomized trials that show that bevacizumab offers an advantage over the optimal fluoropyrimidinecontaining combination chemotherapy regimens (Table 2) [27,47,45,61-63]. However, non-randomized registration trials and cross-study results may lend support for the use of bevacizumab firstline since these studies are sometimes interpreted as giving a signal that bevacizumab improves the efficacy of FOLFIRI (BEAT [64], AVIRI [65]). Besides, in the BICC-C study IFL plus bevacizumab was clearly shown to be inferior to FOLFIRI plus bevacizumab [66,67]. In a recent Phase III trial FOLFIRI plus bevacizumab was compared with FOLFOXIRI plus bevacizumab [51]. FOLFOXIRI plus bevacizumab significantly increased RR and PFS compared with FOLFIRI plus bevacizumab.

However, despite the fact that the FIRE-3 trial failed to meet its primary endpoint, the data

Table 2. Randomized trials of bevacizumab as first-line treatment in metastatic colorectal cancer.

\begin{tabular}{|c|c|c|c|c|}
\hline Regimen & Response rate $^{\dagger}(\%)$ & $\begin{array}{l}\text { Progression-free survival } \\
(\mathrm{HR})^{+} \text {months }\end{array}$ & $\begin{array}{l}\text { Overall survival (HR) } \\
\text { months }\end{array}$ & Ref. \\
\hline 5-FU/LV + bevacizumab (pooled data) & $9.6^{\pi}$ & $3.2\left(0.63^{\natural}\right)$ & $3.3\left(0.74^{\uparrow}\right)$ & [61] \\
\hline IFL + bevacizumab & 3.0 & $2.0(0.86)$ & $3.2(0.82)$ & [45] \\
\hline 5-FU/LV+IRI +/- bevacizumab & 1.6 & NA & $-3.0(\mathrm{NA})$ & [63] \\
\hline XELOX/FOLFOX4 + bevacizumab & $-2.0(0.0)^{\S}$ & $1.4(0.83)$ & $1.4(0.89)$ & [42] \\
\hline CAPOX + bevacizumab & NA & $1.9(0.77)$ & $2.2(0.84)$ & [42] \\
\hline
\end{tabular}


tend to support the use of the treatment sequence FOLFIRI plus cetuximab over FOLFIRI plus bevacizumab as a first-line option for group 3 patients with $R A S$ wild-type disease. The median OS was statistically significantly prolonged from 25.0 to 28.7 months ( $\mathrm{p}=0$ ). As for group 1 patients, group 2 patients with $R A S$ mutant disease could receive combination chemotherapy plus bevacizumab. The median OS of $\sim 25$ months for patients in the bevacizumab arm compared well with the results of other studies, thus it is unlikely that the patients in the bevacizumab arm underperformed in this trial. The results of the CALGB study, however do not confirm those of the FIRE-3 study, even suggesting that FOLFIRI plus bevacizumab may have superior activity to FOLFIRI plus cetuximab, although this was not shown to be statistically significant [30]. Thus, for those group 3 patients with $R A S$ wild-type disease who can tolerate it, combination chemotherapy plus an EGFRtargeting agent (cetuximab or panitumumab) or plus bevacizumab, are treatment possibilities, whilst for those patients with RAS mutant disease chemotherapy plus bevacizumab is probably still the only treatment of choice.

In terms of how long group 3 patients should be treated, data from the NO16966 trial with bevacizumab show that there is a strong benefit to be achieved in terms of PFS if patients are treated until progression. When comparing the pooled PFS 'general' and 'on-treatment' analyses both of the bevacizumab curves separate in the beginning but start to run parallel at around 6 months. This reflects the fact that a large group of patients in the NO16966 trial discontinued treatment at 6 months and were not treated until progression, as recommended in the protocol [68]. For the patients who cannot tolerate the combination chemotherapy, capecitabine and bevacizumab seems to be a good alternative as shown in the STOP and GO [69], CAIRO 3 [70] and AVEX trials [71].

Use of a biological beyond progression is another important issue to be addressed. Is the resistance due to chemotherapy or chemotherapy plus the biological? Data from the BRITE observational study showed bevacizumab increased survival when continued post progression. This was also confirmed in the second-line setting by the results of the randomized prospective controlled TML trial [72], and data from the VELOUR and BEBYP studies [73-75]. However, data on the second-line use of antiangiogenic therapy seems to suggest that those who have not received prior bevacizumab therapy may have a better outcome (E3200 and VELOUR trials) $[74,76]$.

However, it is clear that the therapeutic sequencing for patients with mCRC is still not clearly established and that further studies will be required to determine the optimal sequencing of the chemotherapy plus $\mathrm{mAb}$ therapies in $\mathrm{mCRC}$ patients with $R A S$ wild-type and $R A S$ mutant disease. In fact, the median OS for patients treated in the TML study and calculated from first-line treatment was only 23.8 months, and therefore slightly inferior to the FOLFIRI plus bevacizumab arm and definitely inferior to the FOLFIRI plus cetuximab arm of the FIRE-3 trial, although patients with aggressive tumors (i.e. progression within 3 months of chemotherapy plus bevacizumab, first-line) were excluded from second-line therapy. It should also be kept in mind that $R A S$ testing was not required in the TML study. Thus, it is very unlikely that treatment with bevacizumab up until and beyond progression will achieve similar efficacy to treatment sequences using cetuximab or panitumumab in combination with chemotherapy first-line or bevacizumab in combination with chemotherapy second-line as reported for patients with KRAS and $R A S$ wild-type disease in the FIRE-3 study. Although, it is clear that both the use of bevacizumab up to and beyond progression and the results for bevacizumab in the FIRE-3, PEAK and CALGB studies need to be validated further.

If we consider the treatment sequence further, second-line therapy is normally proposed for patients with good PS and adequate organ function, and is dependent on the first-line therapy choice. For patients in whom the initial chemotherapy backbone has failed the cytotoxic regimen should be changed [8]. Cetuximab has been demonstrated to work in combination with irinotecan second-line in terms of RR [77] and differs from bevacizumab in that it demonstrates efficacy as a monotherapy in second- and thirdline [77-79]. Panitumumab has also been shown to be active second-line in combination with FOLFIRI [80]. Similarly, the new antiangiogenic agent aflibercept which has been approved in Europe and the US in combination with FOLFIRI for the treatment of patients failing prior oxaliplatin therapy, could be introduced at this stage [73]. As mentioned above, a benefit has also been reported for patients receiving prior bevacizumab therapy $[74,81]$. Both cetuximab 
and bevacizumab have also shown efficacy in the third-line/salvage therapy setting [77,82]. The new antiangiogenic regorafenib has also reported salvage activity versus best supportive care [83,84].

Given the lack of clear recommendations [6,85], the advisors were asked to consider the suggestions for the sequencing of biologicals outlined in Table 3 for themselves, based on the ability to select patients according to the $R A S$ mutational status of their disease.

\section{Arriving at a consensus}

It is clear that initial RR is important for group 1 and 2 patients and that OS is important for group 3 patients. The strong recommendation by the experts and advisors was that cetuximab/ panitumumab should be used in combination with standard first-line infusional chemotherapy regimens in the treatment of $\mathrm{mCRC}$ patients with $R A S$ wild-type tumors within the countries represented by the experts, approvals and reimbursement permitting. Doublet chemotherapy combinations or FOLFOXIRI plus/minus bevacizumab may provide an alternative but the latter particularly is likely to be more toxic than FOLFIRI or FOLFOX plus an EGFR-targeted agent for patients with $R A S$ wild-type tumors. The experts agreed that bevacizumab is an option first-line.

There was $100 \%$ agreement among the experts and advisors that the therapeutic strategies for patients with mCRC depended on the treatment goal, patient characteristics, and tumor characteristics as well as predictive and prognostic factors. All advisors agreed that tumor $R A S$ mutational status was the only validated pretreatment biomarker. Indeed it was remarked upon that the presence of a tumor $R A S$ mutation was a stronger negative predictor than tumor $R A S$ wild-type mutation status was a positive predictor. Thus it seems reasonable to recommend the upfront determination of patient tumor RAS (KRAS and $N R A S)$ mutational status for all patients with metastatic disease as a prequel to making any decisions on their first-line therapy. When asked the question, 'which of the endpoints RR, PFS, and OS' is most important?', $33.3 \%$ of voters considered RR to be most important, particularly for symptomatic patients, $50 \%$ considered OS to be important and only $16.7 \%$ considered PFS to be important.

When the advisors were asked if 'based on the currently available data, they accepted that an EGFR-targeted agent in combination with a standard infusional combination chemotherapy regimen offers a significant benefit, compared with chemotherapy alone, across all efficacy endpoints in the first-line treatment of patients with $R A S$ wild-type mCRC', $63.6 \%$ of the participants agreed, $27.3 \%$ conditionally agreed, the remaining $9.1 \%$ (one participant) did not agree. All the advisors after discussion agreed that cetuximab (panitumumab) in combination with a standard infusional combination chemotherapy regimen offered a significant benefit, compared with the same chemotherapy alone, in the first-line treatment of group 1 and 2 patients with $K R A S$ wild-type mCRC.

Of the advisors present, $76.9 \%$ routinely treated their patients within the framework of a MDT and the consensus was that cetuximab plus an infusional combination chemotherapy regimen improves the chance of resection compared with treatment with chemotherapy alone

Table 3. Suggested sequencing of approved targeted agents in the treatment of patients with metastatic colorectal cancer.

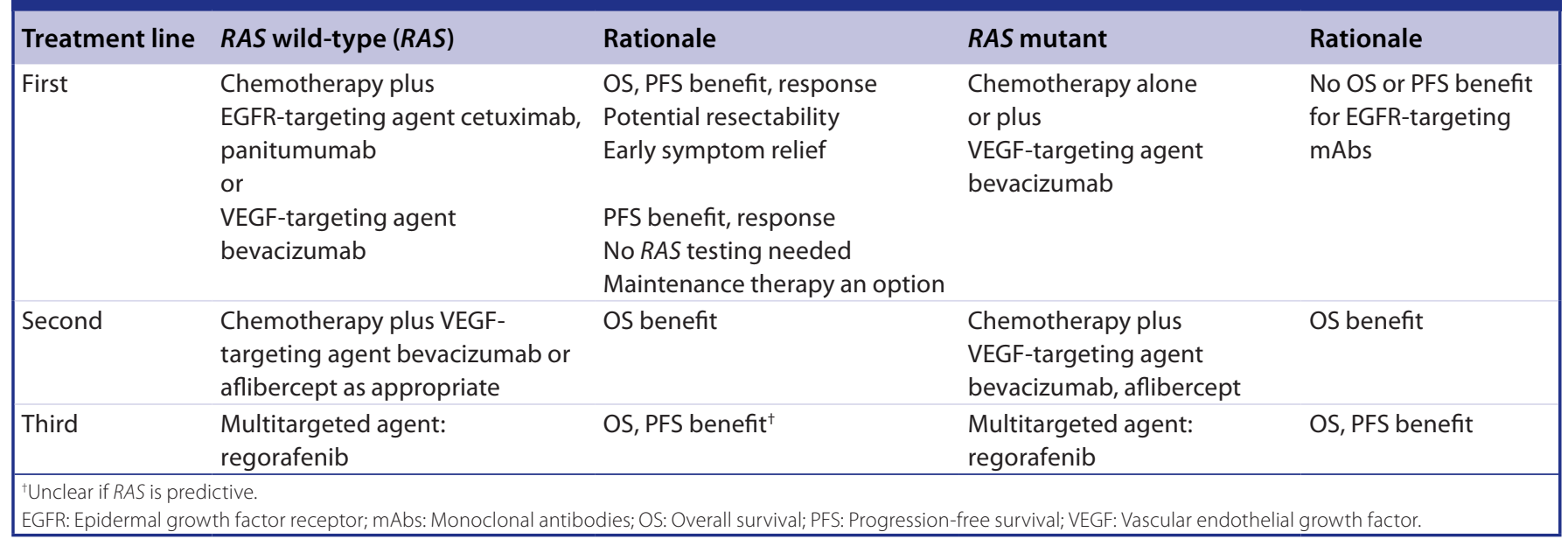


and provides the hope of a cure in patients with initially non-resectable $R A S$ wild-type mCRC. All but one of the participants, agreed that antiEGFR therapy in combination with standard infusional combination chemotherapy confers a benefit for group 1 and $2 R A S$ wild-type patients, but there was uncertainty about whether the EGFR therapies cetuximab and panitumumab were the same. The use of cetuximab or panitumumab in combination with chemotherapy was also supported for group 3 patients with RAS wild-type disease where appropriate. All but one of the participants thought that the choice of chemotherapy backbone was a valid concern.

The consensus was that personalized treatment is better than 'one treatment fits all' in the first-line treatment of patients with $\mathrm{mCRC}$, within the limitations of our current knowledge and evidence base. Furthermore, all advisors believed that personalized medicine was the future. Patient tumor $R A S$ analysis was available to all participating experts.

However, the participants felt that, although cetuximab and panitumumab as EGFR-targeting mAbs were essentially the same class of targeted agent, the National Comprehensive Cancer Network (NCCN) was wrong to make a distinction between cetuximab and panitumumab and recommend FOLFOX plus panitumumab as initial therapy when data are also available for cetuximab plus FOLFOX and to recommend FOLFIRI plus cetuximab or panitumumab when there are no data for panitumumab with FOLFIRI, first line. The regimen in the pivotal study of cetuximab (CRYSTAL) was FOLFIRI whilst it was FOLFOX in the panitumumab pivotal study (PRIME). Cetuximab was investigated in combination with FOLFOX in the randomized OPUS study. It should be noted that these guidelines are due to be updated online in August 2014. Indeed some participants felt that European Society for Medical Oncology (ESMO) was wrong to say that cetuximab and panitumumab were the same, resulting in some guideline recommendations being made in the absence of supporting data. On the other hand however, it was felt very strongly that there were insufficient data to say that cetuximab and panitumumab were different in terms of treatment efficacy.

\section{Summary of the consensus} recommendations

The overall consensus recommendations were that:
- Group 1 patients should be treated within a MDT environment wherever possible;

- Determination of patient tumor RAS mutational status should be carried out for all patients with mCRC prior to deciding their first-line therapy in a step towards more strategic and personalized treatment approaches;

- Cetuximab should be used in combination with the standard infusional chemotherapy regimens FOLFIRI or FOLFOX or panitumumab can be used in combination with FOLFOX in the first-line treatment of $\mathrm{mCRC}$ patients with $R A S$ wild-type tumors for group 1 and 2 patients and even in the treatment of certain group 3 patients as appropriate;

- Patients with RAS wild-type mCRC should receive EGFR $\mathrm{mAb}$-containing first-line therapy whenever possible;

- Although, bevacizumab has recently shown comparable activity in the FIRE-3 (for RR and PFS) and CALGB (for OS) studies;

- Patients with RAS mutant disease or disease of unknown $R A S$ mutation status can be treated with combination chemotherapy plus bevacizumab;

- In relation to second-line therapy, there is evidence that FOLFOX is more active second-line than FOLFIRI [86] and FOLFOX plus bevacizumab has been shown to be active secondline. FOLFIRI plus aflibercept is also another second-line option [73,74]. Furthermore, data from the FIRE-3 trial, presented after the advisory board on which these recommendations are based, support the use of the treatment sequence FOLFIRI plus cetuximab followed by FOLFOX plus bevacizumab in patients with $R A S$ wild-type disease [28]. The recent CALGB data however show that all combinations and sequencing are acceptable [30] and further results from this analysis are awaited;

- All participants endorsed more personalized and strategic treatment approaches.

\section{Future perspective}

Colorectal cancer (CRC) is one of the most commonly diagnosed cancers worldwide. Current recommendations and guidelines propose that patients with initially unresectable metastatic disease (mCRC) should be stratified for first-line treatment by dividing them into three clinical 
groups according to clinical presentation and treatment goal. Increasingly it seems likely that the optimal treatment strategy for all three patient groups is likely to involve a targeted biologic. However, the treatment options for these patients merit further review and refinement in the light not only of the recent evidence that additional tumor $R A S$ mutations, beyond those identified in $K R A S$ exon 2 codons 12/13, predict for a lack of response in patients receiving the EGFR-targeted therapies cetuximab and panitumumab, but also the results of recent head to head studies of cetuximab and panitumumab versus bevacizumab in the first-line treatment

\section{EXECUTIVE SUMMARY}

\section{Introduction \& background}

- One therapeutic approach does not work for all and currently tumor RAS mutation status is used to identify those patients with metastatic colorectal cancer (mCRC) who are most likely to benefit from systemic therapy with EGFRtargeting monoclonal antibody $(\mathrm{mAb})$ therapies.

\section{Optimization: the evidence}

- Patients with unresectable $m C R C$ should be categorized into three groups according to their disease status at presentation, treatment goal, and tumor RAS gene mutational status as a prequel to a more personalized systemic treatment approach.

\section{Management of group 1 patients}

- Patients, with potentially resectable liver and/or lung metastases only, should receive combination chemotherapy plus a targeted agent, whenever possible.

- Patients with RAS wild-type disease should receive an EGFR-targeting mAb plus FOLFIRI/FOLFOX, local regulations and guidance permitting. Chemotherapy (FOLFOX/XELOX/FOLFIRI) plus bevacizumab is also an option.

- Patients with RAS mutant disease should receive combination chemotherapy plus bevacizumab. FOLFOXIRI plus or minus bevacizumab is also an option for patients with either RAS wild-type or mutant disease and may be useful in combination with bevacizumab in patients with BRAF mutant tumors.

\section{Management of group 2 patients}

- For RAS wild-type patients with poor performance status and aggressive symptomatic disease, combinations of cetuximab or panitumumab with a standard infusional combination chemotherapy regimen offer the potential for improved symptom relief over chemotherapy alone. For patients with RAS mutant disease, combination chemotherapy plus bevacizumab could be used. FOLFOXIRI plus or minus bevacizumab may also be an option.

\section{Management of group 3 patients}

- For patients with non-resectable metastases, asymptomatic and less aggressive disease the addition of bevacizumab to fluoropyrimidine therapy may confer a benefit.

- For those group 3 patients with RAS wild-type disease who can tolerate it, combination chemotherapy plus bevacizumab or an EGFR-targeting agent are treatment possibilities.

\section{Summary of the consensus recommendation}

- Patients with potentially resectable liver and/or lung metastases should be treated within a multidisciplinary team environment.

- Cetuximab plus FOLFIRI/FOLFOX or panitumumab plus FOLFOX should be used for the first-line treatment of group 1 and $2 \mathrm{mCRC}$ patients with RAS wild-type tumors and in the treatment of certain group 3 patients whenever possible. Chemotherapy plus bevacizumab is also an option.

- Patients with RAS mutant disease or disease of unknown RAS mutational status should be treated with combination chemotherapy plus bevacizumab.

- An EGFR-targeting agent should be part of a continuum of care for patients with RAS wild-type mCRC within the countries represented, approvals and reimbursement permitting, as part of a more strategic and personalized treatment approach. 
setting which may provide an insight into the use and optimal sequencing of these agents in mCRC patients with $R A S$ wild-type disease.

Thus, even in this era of expanded $R A S$ analysis, where fewer patients are eligible for treatment with EGFR-targeting $\mathrm{mAb}$ therapy, it seems appropriate to conclude from the available data that all mCRC patients should undergo expanded $R A S$ mutational analysis of their tumors and that patients with $R A S$ wildtype $\mathrm{mCRC}$ can be treated with an EGFR mAb first-line as an adjunct to standard infusional combination chemotherapy, to maximize their potential treatment outcomes. Indeed patients with $R A S$ wild-type $\mathrm{mCRC}$ can be treated with both EGFR and VEGF $\mathrm{mAb}$ as a part of continuum of care in first- and second- line settings and patients with $R A S$ mutant mCRC should be treated with an antiangiogenic agent in combination with chemotherapy in all lines. Already, median overall survivals of 30 months are being reported for patients with $\mathrm{mCRC}$ in clinical trials employing strategic therapy approaches. Going forward it is likely that treatment can be tailored to more highly genetically selected patient groups with the strategic sequencing and the 'switching' of therapies augmenting the median overall survival times for these patients.

\section{Financial \& competing interests disclosure}

Regarding potential conflicts of interest in relation to the current study, S Yalcin reported an advisory board role for Roche, Merck Serono, Sanofi, Novartis and Amgen; A Nosworthy reported receiving personal fees and non-financial support from Merck Serono; A Tumanova reported receiving research funding from Merck Serono; $S$ Tejpar reported receiving speaker fees and past research grants from Merck Serono and C-H Köhne reported receiving honoraria from Merck $K G a A$, Amgen and Bayer. Regarding potential conflicts of interest outside the submitted work, H Halawani reported receiving personal fees from Merck Serono; A Meshcheryakov reported receiving personal fees from Merck and Sanofi; A Nosworthy reported receiving personal fees and non-financial support from Bristol Myers Squibb and Roche and personal fees from Lilly; C-H Köhne reported receiving honoraria from Merck KGaA, Amgen and Bayer. Y Abdel Kader, D Trad, OG Demir, R Mall, F Nasr, D Osinsky and NS Turhal declared no conflicts of interest. The authors have no other relevant affiliations or financial involvement with any organization or entity with a financial interest in or financial conflict with the subject matter or materials discussed in the manuscript apart from those disclosed.

The authors would like to thank A Kinsella of Cancer Communications \& Consultancy Ltd (Knutsford, Cheshire, UK) for the provision of medical writing services, funded by Merck KGaA. These included initial drafting of the manuscript, from attendance at the Advisory Board Meeting, and subsequent revision under the guidance of the authors, who had final responsibility for the manuscript content. Merck KGaA exerted no influence on the content of the manuscript.

\section{Open Access}

This work is licensed under the Creative Commons Attribution-NonCommercial 3.0 Unported License. To view a copy of this license, visit http://creativecommons.org/ licenses/by-nc-nd/3.0/

\section{References}

Papers of special note have been highlighted as: - of interest; $\bullet$ of considerable interest

1 Ferlay J, Parkin DM, Steliarova-Foucher E. Estimates of cancer incidence and mortality in Europe in 2008. Eur. J. Cancer 46(4), 765-781 (2010).

2 Ferlay J, Shin HR, Bray F, Forman D, Mathers C, Parkin DM. Estimates of worldwide burden of cancer in 2008: GLOBOCAN 2008. Int. J. Cancer 127(12), 2893-2917 (2010).

3 Kohne $\mathrm{CH}$, Cunningham D, Di Costanzo F et al. Clinical determinants of survival in patients with 5-fluorouracil-based treatment for metastatic colorectal cancer: results of a multivariate analysis of 3825 patients. Ann. Oncol. 13(2), 308-317 (2002).

4 Sanoff HK, Mcleod HL. Predictive factors for response and toxicity in Chemotherapy: pharmacogenomics. Semin. Colon Rectal Surg. 19(4), 226-230 (2008).

5 Diaz R, Aparicio J, Girones R et al. Analysis of prognostic factors and applicability of Kohne's prognostic groups in patients with metastatic colorectal cancer treated with first-line irinotecan or oxaliplatin-based Chemotherapy. Clin. Colorectal Cancer 5(3), 197-202 (2005).

6 Schmoll HJ, Van Cutsem E, Stein A et al. ESMO Consensus Guidelines for management of patients with colon and rectal cancer. a personalized approach to clinical decision making. Ann. Oncol. 23(10), 2479-2516 (2012).

7 Van Cutsem E, Dicato M, Arber N et al. Molecular markers and biological targeted therapies in metastatic colorectal cancer: expert opinion and recommendations derived from the 11th ESMO/World Congress on Gastrointestinal Cancer, Barcelona, 2009. Ann. Oncol. 21(Suppl. 6), vi1-vi10 (2010).

8 Van Cutsem E, Nordlinger B, Cervantes A. Advanced colorectal cancer: ESMO Clinical Practice Guidelines for treatment. Ann. Oncol. 21(Suppl. 5), v93-v97 (2010).

9 Cartwright TH. Treatment decisions after diagnosis of metastatic colorectal cancer. Clin. Colorectal Cancer 11(3), 155-166 (2012).

10 Adam R, De Gramont A, Figueras J et al. The oncosurgery approach to managing liver metastases from colorectal cancer: a multidisciplinary international consensus. Oncologist 17(10), 1225-1239 (2012).

11 Van Cutsem E, Kohne CH, Hitre E et al. Cetuximab and Chemotherapy as initial treatment for metastatic colorectal cancer. N. Engl. J. Med. 360 (14), 1408-1417 (2009). 
- Demonstrates clinical outcome benefit from the addition of cetuximab to FOLFIRI.

12 Van Cutsem E, Kohne CH, Lang I et al. Cetuximab plus irinotecan, fluorouracil, and leucovorin as first-line treatment for metastatic colorectal cancer: updated analysis of overall survival according to tumor $K R A S$ and $B R A F$ mutation status. J. Clin. Oncol. 29(15), 2011-2019 (2011).

- Confirms clinical outcome benefit from the addition of cetuximab to FOLFIRI enhanced in patients with KRAS exon 2 codon 12/13 wild-type tumors.

13 Bokemeyer C, Bondarenko I, Makhson A et al. Fluorouracil, leucovorin, and oxaliplatin with and without cetuximab in the first-line treatment of metastatic colorectal cancer. J. Clin. Oncol. 27(5), 663-671 (2009).

- Demonstrates clinical outcome benefit from the addition of cetuximab to FOLFOX4.

14 Bokemeyer C, Bondarenko I, Hartmann JT et al. Efficacy according to biomarker status of cetuximab plus FOLFOX-4 as first-line treatment for metastatic colorectal cancer: the OPUS study. Ann. Oncol. 22(7), 1535-1546 (2011).

- Confirms clinical outcome benefit from the addition of cetuximab to FOLFOX4 enhanced in patients with $K R A S$ exon 2 codon $12 / 13$ wild-type tumors.

15 Douillard JY, Siena S, Cassidy J et al. Randomized, Phase III trial of panitumumab with infusional fluorouracil, leucovorin, and oxaliplatin (FOLFOX4) versus FOLFOX4 alone as first-line treatment in patients with previously untreated metastatic colorectal cancer: the PRIME study. J. Clin. Oncol. 28(31), 4697-4705 (2010).

- Demonstrates clinical outcome benefit from the addition of panitumumab to FOLFOX4.

16 Bokemeyer C, Van Cutsem E, Rougier P et al. Addition of cetuximab to Chemotherapy as first-line treatment for $K R A S$ wild-type metastatic colorectal cancer: pooled analysis of the CRYSTAL and OPUS randomised clinical trials. Eur. J. Cancer 48(10), 1466-1475 (2012).

17 Bokemeyer C, Kohne C, Ciardiello F et al. Treatment outcome according to tumor $R A S$ mutation status in OPUS study patients with metastatic colorectal cancer (mCRC) randomized to FOLFOX4 with/without cetuximab. J. Clin. Oncol. 32(5s Suppl.), Abstract 3505 (2014).

- Confirms clinical outcome benefit from the addition of cetuximab to FOLFOX4 is enhanced in patients with $K R A S$ exon 2, 3 and 4 and $N R A S$ exon 2, 3 and 4 wild-type tumors: no benefit observed in patients with KRAS exon 2, 3 and 4 and NRAS exon 2, 3, and 4 mutant tumors.

18 Douillard JY, Oliner KS, Siena $S$ et al. Panitumumab-FOLFOX4 treatment and RAS mutations in colorectal cancer. $N$. Engl. J. Med. 369(11), 1023-1034 (2013).

- Confirms clinical outcome benefit from the addition of panitumumab to FOLFOX 4 is enhanced in patients with KRAS exon 2, 3 and 4 and $N R A S$ exon 2, 3 and 4 wild-type tumors: no benefit observed in patients with $K R A S$ exon 2, 3 and 4 and NRAS exon 2, 3 and 4 mutant tumors.

19 Douillard JY, Siena S, Cassidy J et al. Final results from PRIME: randomized Phase 3 study of panitumumab with FOLFOX4 for first-line treatment of metastatic colorectal cancer. Ann. Oncol. 25(7), 1346-1355 (2014).

- Demonstrates clinical outcome benefit from the addition of panitumumab to FOLFOX4.

20 Oliner KS, Douillard J-Y, Siena $S$ et al. Analysis of KRAS/NRAS and BRAF mutations in the Phase III PRIME study of panitumumab (pmab) plus FOLFOX versus FOLFOX as first-line treatment (tx) for metastatic colorectal cancer (mCRC). J. Clin.

Oncol. 31(Suppl.), Abstract 3511 (2013).

21 Ciardiello F, Lenz HJ, Kohne $\mathrm{C}$ et al. Treatment outcome according to tumor RAS mutation status in CRYSTAL study patients with metastatic colorectal cancer (mCRC) randomized to FOLFIRI with/without cetuximab. J. Clin. Oncol. 32(5s, Suppl.), Abstract 3506 (2014).

- Confirms clinical outcome benefit from the addition of cetuximab to FOLFIRI is enhanced in patients with $K R A S$ exon 2, 3 and 4 and $N R A S$ exon 2, 3 and 4 wild-type tumors: no benefit observed in patients with KRAS exon 2, 3 and 4 and NRAS exon 2, 3 and 4 mutant tumors.

22 Piessevaux H, Buyse M, Schlichting M et al. Use of early tumor shrinkage to predict long-term outcome in metastatic colorectal cancer treated with cetuximab. J. Clin. Oncol. 31(30), 3764-3775 (2013).

23 Mansmann UR, Laubender RP, Sartorius U, Giessen CA, Graser A, Heinemann V. Improved early prediction of individual prognosis for patients with mCRC: joint modeling of tumor shrinkage with volume data for PFS and OS. J. Clin. Oncol. 30 (Suppl. 15), Abstract 3603 (2012).

24 Mansmann UR, Sartorius U, Laubender RP, Giessen CA, Esser R, Heinemann V.
Deepness of response: A quantitative analysis of its impact on post-progression survival time after first-line treatment in patients with mCRC. J. Clin. Oncol. 30 (Suppl. 4), Abstract 427 (2012).

25 Maughan TS, Adams RA, Smith CG et al. Addition of cetuximab to oxaliplatin-based first-line combination Chemotherapy for treatment of advanced colorectal cancer: results of the randomised Phase 3 MRC COIN trial. Lancet 377(9783), 2103-2114 (2011).

26 Hurwitz HI, Yi J, Ince W, Novotny WF, Rosen $\mathrm{O}$. The clinical benefit of bevacizumab in metastatic colorectal cancer is independent of K-ras mutation status: analysis of a Phase III study of bevacizumab with Chemotherapy in previously untreated metastatic colorectal cancer. Oncologist 14(1), 22-28 (2009).

27 Cassidy J, Clarke S, Diaz-Rubio E et al. Randomized Phase III study of capecitabine plus oxaliplatin compared with fluorouracil/ folinic acid plus oxaliplatin as first-line therapy for metastatic colorectal cancer. J. Clin. Oncol. 26(12), 2006-2012 (2008).

28 Heinemann V, Fischer Von Weikersthal L, Decker T et al. Randomized comparison of FOLFIRI plus cetuximab versus FOLFIRI plus bevacizumab as first-line treatment of $K R A S$ wild-type metastatic colorectal cancer: German AIO study KRK-0306 (FIRE-3). J. Clin. Oncol. 31(Suppl. 15), Abstract LBA3506 (2013).

- FOLFIRI plus cetuximab superior to FOLFIRI plus bevacizumab first-line in terms of overall survival in patients with $R A S$ wild-type tumors, providing potential insight into optimal sequencing of cetuximab and bevacizumab in patients with $R A S$ wild-type tumors.

29 Schwartzberg LS, Rivera F, Karthaus M et al. PEAK: a randomized, multicenter Phase II study of panitumumab plus modified fluorouracil, leucovorin, and oxaliplatin (mFOLFOX6) or bevacizumab plus mFOLFOX6 in patients with previously untreated, unresectable, wild-type KRAS exon 2 metastatic colorectal cancer. J. Clin. Oncol, 32(21), 2240-2247 (2014).

- Support FIRE-3 data above.

30 Venook A, Niedzwiecki D, Lenz HJ et al. CALGB/SWOG 80405: Phase III trial of irinotecan/5-FU/leucovorin (FOLFIRI) or oxaliplatin/5-FU/leucovorin (mFOLFOX6) with bevacizumab (BV) or cetuximab (CET) for patients (pts) with KRAS wild-type (wt) untreated metastatic adenocarcinoma of the colon or rectum (MCRC). J. Clin. Oncol. 32(5s, Suppl.), Abstract LBA3 (2014). 
31 Van Cutsem E, Nordlinger B, Adam R et al. Towards a pan-European consensus on the treatment of patients with colorectal liver metastases. Eur. J. Cancer 42(14), 2212-2221 (2006).

32 Nordlinger B, Van Cutsem E, Gruenberger T et al. Combination of surgery and chemotherapy and the role of targeted agents in the treatment of patients with colorectal liver metastases: recommendations from an expert panel. Ann. Oncol. 20 (6), 985-992 (2009).

33 Nordlinger B, Van Cutsem E, Rougier P et al. Does Chemotherapy prior to liver resection increase the potential for cure in patients with metastatic colorectal cancer? A report from the European Colorectal Metastases Treatment Group. Eur. J. Cancer 43(14), 2037-2045 (2007).

34 Folprecht G, Grothey A, Alberts S, Raab HR, Kohne CH. Neoadjuvant treatment of unresectable colorectal liver metastases: correlation between tumour response and resection rates. Ann. Oncol. 16(8), 1311-1319 (2005).

35 Van Cutsem E, Bokemeyer C, Heeger S, Sartorius U, Rougier P, Kohne C. Outcome according to metastatic site in patients with $K R A S$ wild-type tumors: analysis from the CRYSTAL and OPUS studies. J. Clin. Oncol. 29(Suppl. 4), Abstract 472 (2011).

36 Kohne C, Bokemeyer C, Heeger S, Sartorius U, Rougier P, Van Cutsem E. Efficacy of Chemotherapy plus cetuximab according to metastatic site in $K R A S$ wild-type metastatic colorectal cancer (mCRC): Analysis of CRYSTAL and OPUS studies. J. Clin. Oncol. 29(Suppl. 15), Abstract 3576 (2011).

37 Ye LC, Liu TS, Ren L et al. Randomized controlled trial of cetuximab plus Chemotherapy for patients with KRAS wild-type unresectable colorectal liver-limited metastases. J. Clin. Oncol. 31(16), 1931-1938 (2013).

38 Primrose J, Falk S, Finch-Jones $\mathrm{M}$ et al. Systemic chemotherapy with or without cetuximab in patients with resectable colorectal liver metastasis: the New EPOC randomised controlled trial. Lancet Oncol. 15(6), 601-611 (2014).

39 Tveit KM, Guren T, Glimelius B et al. Phase III trial of cetuximab with continuous or intermittent fluorouracil, leucovorin, and oxaliplatin (Nordic FLOX) versus FLOX alone in first-line treatment of metastatic colorectal cancer: the NORDIC-VII study. J. Clin. Oncol. 30 (15), 1755-1762 (2012).

40 Ku GY, Haaland BA, De Lima Lopes G Jr. Cetuximab in the first-line treatment of K-ras wild-type metastatic colorectal cancer: the choice and schedule of fluoropyrimidine matters. Cancer Chemother. Pharmacol. 70(2), 231-238 (2012).

41 Arkenau HT, Arnold D, Cassidy J et al. Efficacy of oxaliplatin plus capecitabine or infusional fluorouracil/leucovorin in patients with metastatic colorectal cancer: a pooled analysis of randomized trials. J. Clin. Oncol. 26(36), 5910-5917 (2008).

42 Saltz LB, Clarke S, Diaz-Rubio E et al. Bevacizumab in combination with oxaliplatin-based Chemotherapy as first-line therapy in metastatic colorectal cancer: a randomized Phase III study. J. Clin. Oncol. 26(12), 2013-2019 (2008).

43 Wong NS, Fernando NH, Nixon AB et al. A Phase II study of capecitabine, oxaliplatin, bevacizumab and cetuximab in the treatment of metastatic colorectal cancer. Anticancer Res. 31(1), 255-261 (2011).

44 Gruenberger T, Bridgewater J, Chau I et al. Randomized, Phase II study of bevacizumab with mFOLFOX6 or FOLFOXIRI in patients with initially unresectable liver metastases from colorectal cancer: resectability and safety in OLIVIA. J. Clin. Oncol. 31 (Suppl. 15), Abstract 3619 (2013).

45 Hurwitz H, Fehrenbacher L, Novotny W et al. Bevacizumab plus irinotecan, fluorouracil, and leucovorin for metastatic colorectal cancer. N. Engl. J. Med. 350 (23), 2335-2342 (2004).

46 Folprecht G, Gruenberger T, Bechstein W et al. Cetuximab and Chemotherapy in the treatment of patients with initially "nonresectable" colorectal (CRC) liver metastases: long-term follow-up of the CELIM trial. J. Clin. Oncol. 31(Suppl. 15), Abstract 3538 (2013).

47 Suzuki C, Blomqvist L, Sundin A et al. The initial change in tumor size predicts response and survival in patients with metastatic colorectal cancer treated with combination Chemotherapy. Ann. Oncol. 23(4), 948-954 (2012)

48 Mansmann UR, Laubender RP, Giessen CA, Sartorius U, Heinemann V. Validating the prognostic relevance of initial change in tumor size using a series of therapeutic regimens for patients with metastatic colorectal cancer (mCRC). J. Clin. Oncol. 30(Suppl. 4), Abstract 580 (2012).

49 Piessevaux H, Buyse M, De Roock W et al. Radiological tumor size decrease at week 6 is a potent predictor of outcome in chemorefractory metastatic colorectal cancer treated with cetuximab (BOND trial). Ann. Oncol. 20(8), 1375-1382 (2009).

50 Piessevaux H, Van Cutsem E, Bokemeyer C, Schlichting M, Heeger S, Tejpar S. Early tumor shrinkage and long-term outcome in metastatic colorectal cancer (mCRC): assessment of predictive utility across treatment arms in the CRYSTAL and OPUS studies. J. Clin. Oncol. 29(Suppl. 15), Abstract 3572 (2011).

51 Falcone A, Cremolini C, Masi G et al. FOLFOXIRI/bevacizumab (bev) versus FOLFIRI/bev as first-line treatment in unresectable metastatic colorectal cancer (mCRC) patients (pts): results of the Phase III TRIBE trial by GONO group. J. Clin. Oncol. 31(Suppl. 15), Abstract 3505 (2013).

52 Mansmann UR, Sartorius U, Laubender RP, Giessen CA, Esser R, Heinemann V. Deepness of Response: a quantitative analysis of its impact on post-progression survival time after first-line treatment in patients with mCRC. J. Clin. Oncol. 30 (Suppl. 4), Abstract 427 (2013).

53 Mansmann UR, Sartorius U, Laubender RP, Giessen CA, Esser R, Heinemann V. Quantitative analysis of the impact of deepness of response on post-progression survival time following first-line treatment in patients with mCRC. J. Clin. Oncol. 31(Suppl.), Abstract 3630 (2013).

54 Mansmann UR, Sartorius U, Laubender RP, Giessen CA, Esser R, Heinemann V. Quantitative analysis of the impact of deepness of response on post-progression survival time following first-line treatment in patients with mCRC. Ann. Oncol (Suppl.), Abstract O-0009 (2013).

55 Stintzing S, Jung A, Rossius L et al. Analysis of KRAS/NRAS and BRAF mutations in FIRE-3: a randomized Phase III study of FOLFIRI plus cetuximab or bevacizumab as first-line treatment for wild-type (WT) KRAS (exon 2) metastatic colorectal cancer (mCRC) patients. European Cancer Congress 2013, Brussels, Belgium, Abstract 17 (2013).

56 Heinemann V, Modest DP, Fischer Von Weikersthal L et al. Independent radiological evaluation of objective response early tumor shrinkage and depth of response in FIRE-3 (AIO KRK-0306). Ann. Oncol. 25(Suppl. 2), Abstract O-0030 (2014).

57 Falcone A, Ricci S, Brunetti I et al. Phase III trial of infusional fluorouracil, leucovorin, oxaliplatin, and irinotecan (FOLFOXIRI) compared with infusional fluorouracil, leucovorin, and irinotecan (FOLFIRI) as first-line treatment for metastatic colorectal cancer: the Gruppo Oncologico Nord Ovest. J. Clin. Oncol. 25(13), 1670-1676 (2007).

58 Loupakis F, Cremolini C, Salvatore L et al. FOLFOXIRI plus bevacizumab as first-line treatment in BRAF mutant metastatic colorectal cancer. Eur. J. Cancer 50(1), 57-63 (2014). 
59 Goldberg RM, Kohne CH, Seymour MT et al. A pooled safety and efficacy analysis examining the effect of performance status (PS) on outcomes in nine first-line treatment (rx) trials (cts) of 6,286 patients (pts) with metastatic colorectal cancer (mCRC). J. Clin. Oncol. 25(18S), Abstract 4011 (2007).

60 Lang I, Kohne CH, Folprecht G et al. Quality of life analysis in patients with KRAS wild-type metastatic colorectal cancer treated first-line with cetuximab plus irinotecan, fluorouracil and leucovorin. Eur. J. Cancer 49(2), 439-448 (2013).

61 Kabbinavar FF, Hambleton J, Mass RD, Hurwitz HI, Bergsland E, Sarkar S. Combined analysis of efficacy: the addition of bevacizumab to fluorouracil/leucovorin improves survival for patients with metastatic colorectal cancer. J. Clin. Oncol. 23(16), 3706-3712 (2005).

62 Tebbutt NC, Wilson K, Gebski VJ et al. Capecitabine, bevacizumab, and mitomycin in first-line treatment of metastatic colorectal cancer: results of the Australasian gastrointestinal trials group randomized Phase III MAX study. J. Clin. Oncol. 28(19), 3191-3198 (2010).

63 Stathopoulos GP, Batziou C, Trafalis D et al. Treatment of colorectal cancer with and without bevacizumab: a Phase III study. Oncology 78(5-6), 376-381 (2010).

64 Van Cutsem E, Rivera F, Berry S et al. Safety and efficacy of first-line bevacizumab with FOLFOX, XELOX, FOLFIRI and fluoropyrimidines in metastatic colorectal cancer: the BEAT study. Ann. Oncol. 20 (11), 1842-1847 (2009).

65 Sobrero A, Ackland S, Clarke S et al. Phase IV study of bevacizumab in combination with infusional fluorouracil, leucovorin and irinotecan (FOLFIRI) in first-line metastatic colorectal cancer. Oncology 77(2), 113-119 (2009).

66 Fuchs CS, Marshall J, Barrueco J. Randomized, controlled trial of irinotecan plus infusional, bolus, or oral fluoropyrimidines in first-line treatment of metastatic colorectal cancer: updated results from the BICC-C study. J. Clin. Oncol. 26(4), 689-690 (2008).

67 Fuchs CS, Marshall J, Mitchell E et al. Randomized, controlled trial of irinotecan plus infusional, bolus, or oral fluoropyrimidines in first-line treatment of metastatic colorectal cancer: results from the BICC-C Study. J. Clin. Oncol. 25(30), 4779-4786 (2007).

68 Saltz L, Clarke S, Diaz-Rubio E et al. Bevacizumab (Bev) in combination with XELOX or FOLFOX4: updated efficacy results from XELOX-1/ NO16966, a randomized
Phase III trial in first-line metastatic colorectal cancer. J. Clin. Oncol. 25(Suppl. 18), Abstract 4028 (2007).

Yalcin S, Uslu R, Dane F et al. Bevacizumab + capecitabine as maintenance therapy after initial bevacizumab + XELOX treatment in previously untreated patients with metastatic colorectal cancer: Phase III 'Stop and Go' study results--a Turkish Oncology Group Trial. Oncology 85(6), 328-335 (2013).

70 Koopman M, Simkens L, May AM, Mol L, Van Tinteren H, Punt CJA. Final results and subgroup analyses of the Phase 3 CAIRO3 study: maintenance treatment with capecitabine + bevacizumab versus observation after induction treatment with Chemotherapy + bevacizumab in metastatic colorectal cancer (mCRC). J. Clin. Oncol. 32(Suppl. 5), Abstract 3504 (2014).

71 Cunningham D, Lang I, Marcuello E et al. Bevacizumab plus capecitabine versus capecitabine alone in elderly patients with previously untreated metastatic colorectal cancer (AVEX): an open-label, randomised Phase 3 trial. Lancet Oncol. 14(11), 10771085 (2013).

72 Bennouna J, Sastre J, Arnold D et al. Continuation of bevacizumab after first progression in metastatic colorectal cancer (ML18147): a randomised Phase 3 trial. Lancet Oncol. 14(1), 29-37 (2013).

73 Van Cutsem E, Tabernero J, Lakomy R et al. Addition of aflibercept to fluorouracil, leucovorin, and irinotecan improves survival in a Phase III randomized trial in patients with metastatic colorectal cancer previously treated with an oxaliplatin-based regimen. J. Clin. Oncol. 30(28), 3499-3506 (2012).

74 Tabernero J, Van Cutsem E, Lakomy R et al. Results from VELOUR, a Phase 3 study of aflibercept (A) versus placebo (pbo) in combination with FOLFIRI for the treatment of patients (pt) with previously treated metastatic colorectal cancer (MCRC). Eur. J. Cancer 47(Suppl. 2), 5 (2011).

75 Masi G, Loupakis F, Salvatore L et al. Second-line chemotherapy (CT) with or without bevacizumab (BV) in metastatic colorectal cancer (mCRC) patients (pts) who progressed to a first-line treatment containing BV: updated results of the Phase III "BEBYP" trial by the Gruppo Oncologico Nord Ovest (GONO). J. Clin. Oncol. 31(Suppl. 15), Abstract 3615 (2013).

76 Giantonio BJ, Catalano PJ, Meropol NJ et al. Bevacizumab in combination with oxaliplatin, fluorouracil, and leucovorin (FOLFOX4) for previously treated metastatic colorectal cancer: results from the Eastern Cooperative Oncology Group Study E3200. J. Clin. Oncol. 25(12), 1539-1544 (2007).

77 Cunningham D, Humblet Y, Siena S et al. Cetuximab monotherapy and cetuximab plus irinotecan in irinotecan-refractory metastatic colorectal cancer. N. Engl. J. Med. 351(4), 337-345 (2004).

78 Jonker DJ, O'Callaghan CJ, Karapetis CS et al. Cetuximab for the treatment of colorectal cancer. N. Engl. J. Med. 357(20), 2040-2048 (2007).

79 Karapetis CS, Khambata-Ford S, Jonker DJ et al. K-ras mutations and benefit from cetuximab in advanced colorectal cancer. N. Engl. J. Med. 359 (17), 1757-1765 (2008).

80 Peeters M, Price TJ, Cervantes A et al. Randomized Phase III study of panitumumab with fluorouracil, leucovorin, and irinotecan (FOLFIRI) compared with FOLFIRI alone as second-line treatment in patients with metastatic colorectal cancer. J. Clin. Oncol. 28(31), 4706-4713 (2010).

81 Allegra CJ, Lakomy R, Tabernero J et al. Effects of prior bevacizumab (B) use on outcomes from the VELOUR study: a Phase III study of aflibercept (Afl) and FOLFIRI in patients (pts) with metastatic colorectal cancer (mCRC) after failure of an oxaliplatin regimen. J. Clin. Oncol. 30 (Suppl. 15), Abstract 3505 (2012).

82 Geva R, Vecchione L, Tejpar S, Piessevaux H, Van Cutsem E, Prenen H. Bevacizumab plus chemotherapy as salvage treatment in chemorefractory patients with metastatic colorectal cancer. Onco Targets Ther. 6, 53-58 (2013).

83 Davis SL, Eckhardt SG, Messersmith WA, Jimeno A. The development of regorafenib and its current and potential future role in cancer therapy. Drugs Today (Barc) 49(2), 105-115 (2013).

84 Grothey A, Van Cutsem E, Sobrero A et al. Regorafenib monotherapy for previously treated metastatic colorectal cancer (CORRECT): an international, multicentre, randomised, placebo-controlled, Phase 3 trial. Lancet 381(9863), 303-312 (2013).

85 Benson AB 3rd, Bekaii-Saab T, Chan E et al. Metastatic colon cancer, version 3.2013: featured updates to the NCCN Guidelines. J. Natl Compr. Canc. Netw. 11(2), 141-152 (2013).

86 Tournigand C, Andre T, Achille E et al. FOLFIRI followed by FOLFOX6 or the reverse sequence in advanced colorectal cancer: a randomized GERCOR study. J. Clin. Oncol. 22(2), 229-237 (2004). 